\title{
Gene Expression Patterns Underlying the Reinstatement of Plasticity in the Adult Visual System
}

\author{
Ettore Tiraboschi, ${ }^{1,2}$ Ramon Guirado, ${ }^{1}$ Dario Greco, ${ }^{3}$ Petri Auvinen, ${ }^{4}$ \\ Jose Fernando Maya-Vetencourt, ${ }^{5,6}$ Lamberto Maffei, $^{7}$ and Eero Castrén ${ }^{1}$ \\ ${ }^{1}$ Neuroscience Centre, University of Helsinki, 00790 Helsinki, Finland \\ ${ }^{2}$ SARS Institute, University of Bergen, 5020 Bergen, Norway \\ ${ }^{3}$ Finnish Institute of Occupational Health, 00250 Helsinki, Finland \\ ${ }^{4}$ Institute of Biotechnology, University of Helsinki, 00790 Helsinki, Finland \\ ${ }^{5}$ Centre for Nanotechnology Innovation, Italian Institute of Technology, 56127 Pisa, Italy \\ ${ }^{6}$ Centre for Neuroscience and Cognitive Systems, Italian Institute of Technology, 38068 Rovereto, Italy \\ ${ }^{7}$ Neuroscience Institute, CNR, 56100 Pisa, Italy \\ Correspondence should be addressed to Eero Castrén; eero.castren@helsinki.fi
}

Received 1 May 2013; Accepted 10 June 2013

Academic Editor: Alessandro Sale

Copyright (C) 2013 Ettore Tiraboschi et al. This is an open access article distributed under the Creative Commons Attribution License, which permits unrestricted use, distribution, and reproduction in any medium, provided the original work is properly cited.

\begin{abstract}
The nervous system is highly sensitive to experience during early postnatal life, but this phase of heightened plasticity decreases with age. Recent studies have demonstrated that developmental-like plasticity can be reactivated in the visual cortex of adult animals through environmental or pharmacological manipulations. These findings provide a unique opportunity to study the cellular and molecular mechanisms of adult plasticity. Here we used the monocular deprivation paradigm to investigate largescale gene expression patterns underlying the reinstatement of plasticity produced by fluoxetine in the adult rat visual cortex. We found changes, confirmed with RT-PCRs, in gene expression in different biological themes, such as chromatin structure remodelling, transcription factors, molecules involved in synaptic plasticity, extracellular matrix, and excitatory and inhibitory neurotransmission. Our findings reveal a key role for several molecules such as the metalloproteases Mmp2 and Mmp9 or the glycoprotein Reelin and open up new insights into the mechanisms underlying the reopening of the critical periods in the adult brain.
\end{abstract}

\section{Introduction}

Use-dependent plasticity shapes neuronal networks within sensory systems during early life to optimally represent sensory stimuli [1]. Experience-dependent organization of eye-specific inputs is a major mechanism whereby refinement of synaptic connectivity is achieved in the developing visual system [2-4]. Monocular deprivation during development leads to a loss of cortical connectivity of the deprived eye resulting in a shift of the ocular dominance in the visual cortex, which will become permanent if the MD persists to adulthood [5, 6]. Although neuronal plasticity of the developing brain gradually decreases with age [7], recent findings suggest that it can be reactivated in the adult visual cortex [8] and other regions, such as the amygdala [9]. A variety of experimental manipulations, including enzymatic treatments [10, 11], environmental enrichment [12-15], food restriction [16], genetic manipulations [17, 18], and other manipulations, promote this kind of plasticity [19-21].

Although the mechanisms behind the adult induced plasticity are still unclear, we are beginning to understand the key factors involved. For example, the developmental maturation of neuronal inhibition, mainly through the parvalbumin containing interneurons $[22,23]$, is known to be involved in both the opening and the closure of the critical period [20]. Several extracellular matrix components, such as PSANCAM or the perineuronal nets, have been shown to play a role in the maturation of the inhibitory circuitries and 


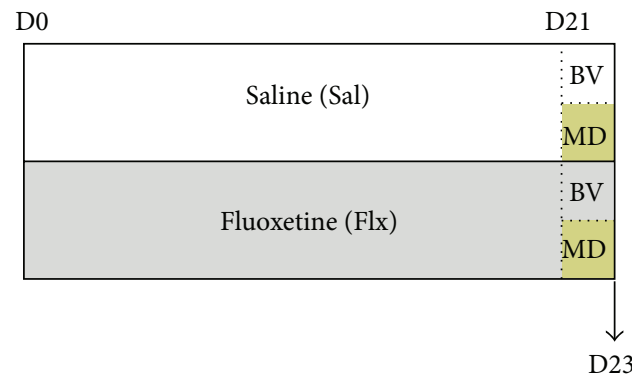

(a)

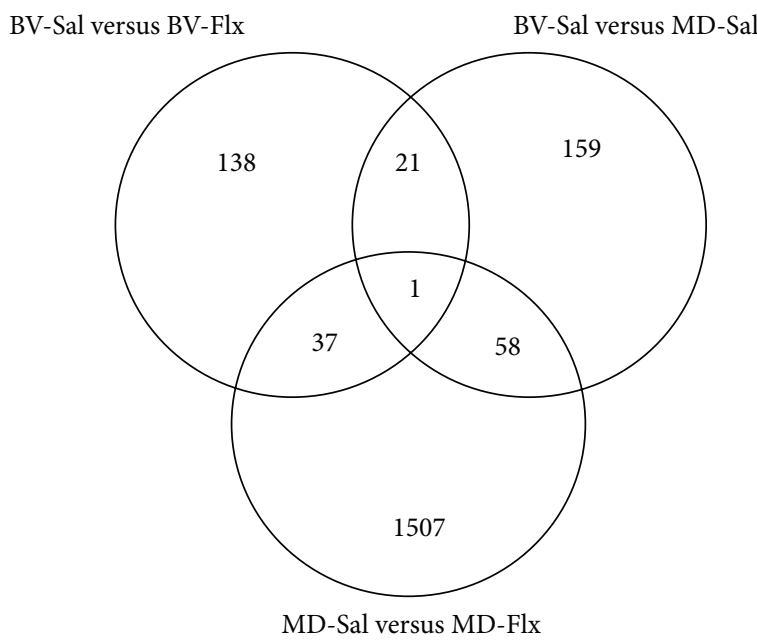

(b)

Figure 1: Experimental design and Venn diagram. (a) Schematic graph showing the different animal groups and experimental conditions conducted. (b) Venn diagram showing the number of genes differentially expressed when comparing the effects of monocular deprivation (BV-Sal versus MD-Sal), the effects of fluoxetine in animals with binocular vision (BV-Sal versus BV-Flx), and those of fluoxetine in animals with monocular deprivation (MD-Sal versus MD-Flx).

experimental manipulations removing these extracellular matrix components, can trigger an early closure [24] or a reopening of the critical period, respectively [11]. Similarly, a variety of other molecules, such as transcription factors [25] or proteins involved in chromatin structure remodeling [26], are also key factors in regulating the closure and reopening of the critical period.

The main pharmacological approaches to experimentally regulate critical period plasticity in the adulthood are those affecting the action of ascending projection systems, such as the serotoninergic or cholinergic systems [21, 27-29]. In this line, we have investigated the plastic effects of antidepressants, such as fluoxetine, that modulate serotoninergic transmission, and we have shown that these drugs, in a longterm treatment, are able to trigger critical period plasticity in the adult brain [8], through an early epigenetic modification that regulates gene expression [29].

Here, we have used fluoxetine in combination with an experience-dependent paradigm of visual deprivation, to analyze the large-scale gene expression patterns, to understand the temporal-dependent changes that allow the reopening of the critical periods in the adult brain.

\section{Experimental Procedures}

2.1. Animal Treatment, Fluoxetine Administration, and Surgical Procedures. A total amount of 32 Long-Evans hooded rats were used in this study, equally distributed in 4 experimental groups ( $n=8$ animals per group), as explained later (Figure 1(a)). Animals were group-housed under standard conditions with food and water ad libitum in plexiglas cages $(40 \times 30 \times 20 \mathrm{~cm})$ and kept in a 12:12 light/dark cycle. Adult rats at the postnatal day 70 (P70) were systemically treated with fluoxetine (fluoxetine-hydrochloride, $0.2 \mathrm{mg} / \mathrm{mL}$ drinking water) for 23 days. Control animals were housed under the same standard conditions drinking tap water.

Three weeks after the beginning of the fluoxetine treatment, rats were anaesthetized with avertin $(1 \mathrm{~mL} / 100 \mathrm{~g})$ and mounted on a stereotaxic apparatus to perform the eyelid suture for monocular deprivation (MD). Eyelids were inspected daily until complete cicatrisation; subjects with even minimal spontaneous reopening were excluded. Great care was taken during the first days after MD to prevent inflammation or infection of the deprived eye through topical application of antibiotic and cortisone.

2.2. DNA Microarrays and Data Analysis. Two days after $\mathrm{MD}$, the binocular region of the primary visual cortex was dissected. For all microarray experiments, total RNA was purified using RNA extraction kit (Macherey Nagel), and Amino Allyl cRNA labeling Kit (Ambion) was used to label cRNA according to manufacturer's standard protocols. Agilent Whole Rat Genome Microarray Kits $(4 \times 44 \mathrm{~K})$ were hybridized following provided protocols.

Images from hybridized microarrays were segmented and the median intensity of each spot was estimated by the software GenePix v.5.0 (axon). Data was imported into the software (http://cran.r-project.org/) and preprocessed by the bioconductor package limma. The statistical analysis used was a linear model followed by $t$-test for finding the differentially expressed genes. In order to increase the reliability of the statistical analysis, we only considered significant those genes with a $P$ value less than 0.01 . In addition, we also increase the reliability of the analysis through validation of the results using multiple RT-PCRs. Lists of significant genes were screened by the DAVID 6.7 annotation tools in order to find overrepresented biological themes. Default DAVID parameters were used. 
2.3. Real-Time PCR. RNA purification was performed according to the standard Trizol procedure (Invitrogen). Purified RNA was treated with DNAse (Fermentas) and cDNA was synthesised from $1 \mu \mathrm{g}$ of RNA (Invitrogen). Real-time PCR was carried out to determine relative enrichment in the samples using the Sybr Green method according to the manufacturer instructions (SYBR Green I master, Light cycler 480, Roche Diagnostics). The comparative Ct method [30] was used to determine the normalized changes of the target gene relative to a calibrator reference; in particular, values were normalized to GAPDH levels. As calibrator reference we referred to Ct from water-treated animal samples.

\section{Results}

3.1. DNA Microarrays. Previous studies have shown that 7 days period of monocular deprivation in fluoxetine-treated adult rats is sufficient to bring about a change in the ocular dominance. To reveal early transcriptional changes that precede and underlie the functional change, we analysed gene expression, using DNA microarrays, at two days after MD. Microarray analysis revealed only relatively few genes that were significantly regulated by either FLX $(n=197$, see Supplementary Table S1 in the Supplementary Material available online at http://dx.doi.org/10.1155/2013/605079) or MD alone $(n=239$, Table S2), treatments that themselves do not produce any changes in the ocular dominance plasticity. However, the combination of FLX and MD, the treatment that promotes changes in ocular dominance, altered the expression of a significantly larger number of genes $(n=$ 1603, Table S3, Figure 1(b)). Notably 1237 out of 1603 (77\%) of the genes in the group receiving both MD and FLX were downregulated, whereas in the groups receiving either MD or FLX, 111 out of 239 and 88 out of 197 genes were downregulated, respectively, comprising of roughly $50 \%$ of all the regulated genes. Hence, the combination of the treatments apparently has two major effects on gene expression; first, it increases the number of regulated genes when compared to the single treatments, and second, it has a striking effect on downregulating most of the genes, indicating that silencing of genes normally expressed during basal conditions is likely involved in the triggering of plasticity of the adult brain.

The representation of biological themes was screened using Fisher's Exact test on the lists of differentially expressed genes in each comparison. Chronic fluoxetine treatment induced a regulation of genes related to chromatin remodelling, nervous system development, and plasticity, as well as regulation of gene expression and transcription in the binocular visual cortex (Table S4).

$\mathrm{MD}$ altered the expression of a significant number of genes related to cell differentiation, cell plasticity, and neurogenesis. Several genes of the ion homeostasis and regulation of transcription were also found overexpressed (Table S5).

The combination of MD and fluoxetine treatment downregulated the majority of the differentially expressed genes, altering the expression of genes represented in a variety of functional processes, including genes related to neuronal development, plasticity, and apoptosis. In addition, genes involved in the synaptic transmission, ion and intracellular calcium homeostasis, and vesicular secretion were found differentially expressed. Blood circulation and lipid metabolism were among the most significantly overrepresented families (Table S6).

3.2. RT-PCR. To provide validation of the microarray data, we next examined single patterns of gene expression by means of real-time PCR, in the same experimental groups and using the same experimental paradigm (Figure 1(a)). In particular, we focused our attention on genes whose expression may alter molecular and cellular processes involved in the closure of the critical period for visual cortex plasticity, such as the balance of inhibitory and excitatory transmission $[22,31,32]$, transcription factors regulating gene expression [25], extracellular matrix remodeling [11], myelination [33], and chromatin structure remodeling [26, 29], as well as genes involved in processes of synaptic plasticity, neuronal differentiation, and outgrowth (see Table 1).

3.2.1. Inhibitory Neurotransmission. We observed that fluoxetine produced a significant increase in the expression of genes involved in inhibitory neurotransmission when comparing both animals with binocular vision and animals with monocular deprivation with their respective controls (BVSal versus BV-Flx and MD-Sal versus MD-Flx; Figure 2(a)). Specifically, in animals with binocular vision, we found an increased expression of the vesicular GABA transporter (VGAT; 60\% increased expression; $P=0.001$ ), while in rats with monocular deprivation together with fluoxetine treatment, we observed an increase in the expression of GABRA4 (30\% increased expression; $P=0.02)$.

3.2.2. Excitatory Neurotransmission. We did not observe many changes in the composition of NMDA receptor subunits in either of the experimental groups (see Figure 2(b) and Table 1). The only significant change we found was a decrease in the expression of the NR2A subunit (NMDA-2A; $20 \%$ decreased expression; $P=0.04$ ) in the animals with monocular deprivation treated with fluoxetine.

3.2.3. Transcription Factors. We detected increases in the gene expression of transcription factors in the animals with binocular vision treated with fluoxetine (Figure 2(c)). In particular, NFKB1 and DLX1 increased their expression $(50 \%$ and $30 \%$ increased expression, resp.; $P=0.04$ and 0.03 , resp.). However, in those animals with monocular deprivation, fluoxetine treatment produced a decrease in the expression of transcription factors, such as EGR-2 $(P=0.04)$.

3.2.4. Synaptic Plasticity. The expression of Reelin, transcript that encodes a glycoprotein that mediates synaptic plasticity at hippocampal level [34], was significantly increased in MD animals treated with fluoxetine (Figure 2(d); 40\% increased expression; $P=0.001)$. The expression of additional transcripts that encode proteins involved in neuronal differentiation and outgrowth processes as well as synaptic plasticity increased in both groups. In animals with binocular 
TABLE 1: RT-PCR analysis.

\begin{tabular}{|c|c|c|c|c|}
\hline Gene symbol & Entrez gene ID & Treatment comparison & $P$ value & Fold change \\
\hline KCNQ3 & 29682 & BV-Sal versus BV-Flx & 0.01 & 1.3 \\
\hline KCNQ3 & 29682 & MD-Sal versus MD-Flx & 0.02 & 1.3 \\
\hline MMP9 & 81687 & BV-Sal versus BV-Flx & 0.13 & 1.1 \\
\hline MMP9 & 81687 & MD-Sal versus MD-Flx & 0.01 & 1.5 \\
\hline VGAT & 83612 & BV-Sal versus BV-Flx & 0.001 & 1.6 \\
\hline VGAT & 83612 & MD-Sal versus MD-Flx & 0.72 & 1.1 \\
\hline DLX1 & 296500 & BV-Sal versus BV-Flx & 0.03 & 1.3 \\
\hline DLX1 & 296500 & MD-Sal versus MD-Flx & 0.25 & 1.0 \\
\hline EGR2 & 114090 & BV-Sal versus BV-Flx & 0.86 & 1.1 \\
\hline EGR2 & 114090 & MD-Sal versus MD-Flx & 0.04 & 0.7 \\
\hline EGR4 & 25129 & BV-Sal versus BV-Flx & 0.29 & 1.2 \\
\hline EGR4 & 25129 & MD-Sal versus MD-Flx & 0.38 & 0.9 \\
\hline mGluR1 & 24414 & BV-Sal versus BV-Flx & 0.47 & 1.0 \\
\hline mGluR1 & 24414 & MD-Sal versus MD-Flx & 0.98 & 1.1 \\
\hline HDAC3 & 15183 & BV-Sal versus BV-Flx & 0.02 & 1.3 \\
\hline HDAC3 & 15183 & MD-Sal versus MD-Flx & 0.92 & 1.1 \\
\hline KCNV1 & 60326 & BV-Sal versus BV-Flx & 0.02 & 1.2 \\
\hline KCNV1 & 60326 & MD-Sal versus MD-Flx & 0.10 & 1.2 \\
\hline NFKB1 & 81736 & BV-Sal versus BV-Flx & 0.04 & 1.5 \\
\hline NFKB1 & 81736 & MD-Sal versus MD-Flx & 0.52 & 1.0 \\
\hline CLCN3 & 84360 & BV-Sal versus BV-Flx & 0.04 & 1.2 \\
\hline CLCN3 & 84360 & MD-Sal versus MD-Flx & 0.01 & 1.5 \\
\hline NR1 & 24408 & BV-Sal versus BV-Flx & 0.45 & 0.9 \\
\hline NR1 & 24408 & MD-Sal versus MD-Flx & 0.43 & 1.1 \\
\hline NR2A & 14811 & BV-Sal versus BV-Flx & 0.63 & 1.1 \\
\hline NR2A & 14811 & MD-Sal versus MD-Flx & 0.04 & 0.8 \\
\hline NR2B & 24410 & BV-Sal versus BV-Flx & 0.51 & 1.1 \\
\hline NR2B & 24410 & MD-Sal versus MD-Flx & 0.07 & 0.8 \\
\hline GABRA1 & 29705 & BV-Sal versus BV-Flx & 0.34 & 1.1 \\
\hline GABRA1 & 29705 & MD-Sal versus MD-Flx & 0.27 & 0.9 \\
\hline GABRA4 & 140675 & BV-Sal versus BV-Flx & 0.29 & 1.1 \\
\hline GABRA4 & 140675 & MD-Sal versus MD-Flx & 0.02 & 1.3 \\
\hline Reelin & 24718 & BV-Sal versus BV-Flx & 0.15 & 1.2 \\
\hline Reelin & 24718 & MD-Sal versus MD-Flx & 0.001 & 1.4 \\
\hline MMP2 & 17390 & BV-Sal versus BV-Flx & 0.02 & 0.5 \\
\hline MMP2 & 17390 & MD-Sal versus MD-Flx & 0.01 & 1.6 \\
\hline $\mathrm{MBP}$ & 24547 & BV-Sal versus BV-Flx & 0.01 & 0.6 \\
\hline MBP & 24547 & MD-Sal versus MD-Flx & 0.01 & 0.6 \\
\hline
\end{tabular}

vision, fluoxetine promoted an increase in the expression of CLCN3 (20\% increased), KCNV1 (20\% increased), and KCNQ3 (30\% increased), which encode ion channels that mediate chloride and potassium conductance $(P<0.05)$, and in animals with monocular deprivation fluoxetine produced also an increase in the expression of CLCN3 (50\% increased expression; $P=0.01$ ).

3.2.5. Extracellular Matrix. The expression of MMP2 and MMP9 was markedly changed between animals treated with fluoxetine and with binocular vision and those with monocular deprivation (Figure 2(e)). MMP2 and MMP9 encode for proteolytic enzymes that degrade extracellular matrix components [35-37] and play a key role in mediating synaptic plasticity at the level of the hippocampus [38, 39]. In particular, MMP2 gene expression was decreased in animals treated with fluoxetine alone (50\% decrease; $P=0.02)$, while animals with combined monocular deprivation and chronic fluoxetine treatment had an increased expression of both MMP2 (60\% increased; $P=0.01)$ and MMP9 $(50 \%$ increased; $P=0.01)$. 


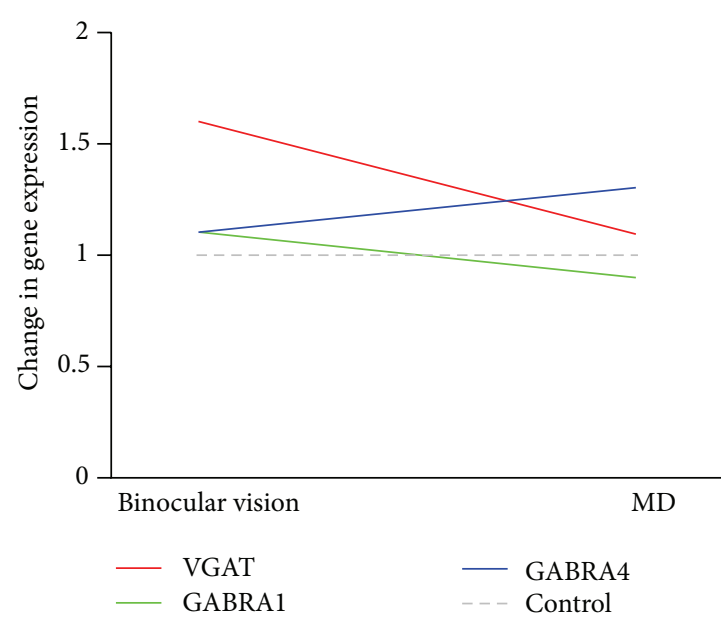

(a) Inhibitory neurotransmission

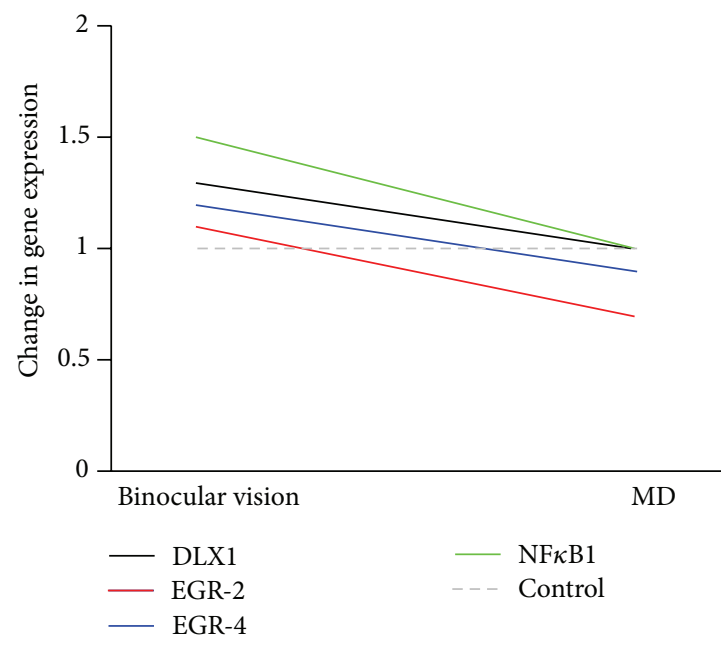

(c) Transcription factors

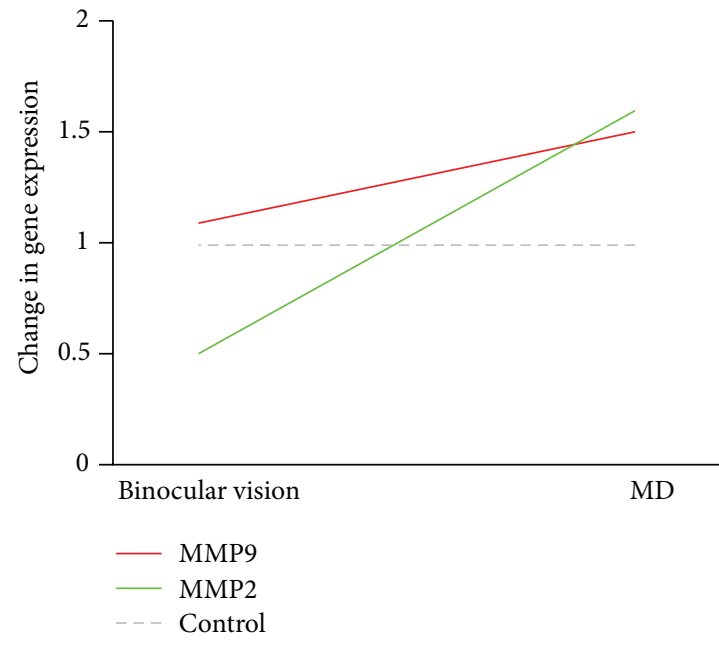

(e) Extracellular matrix

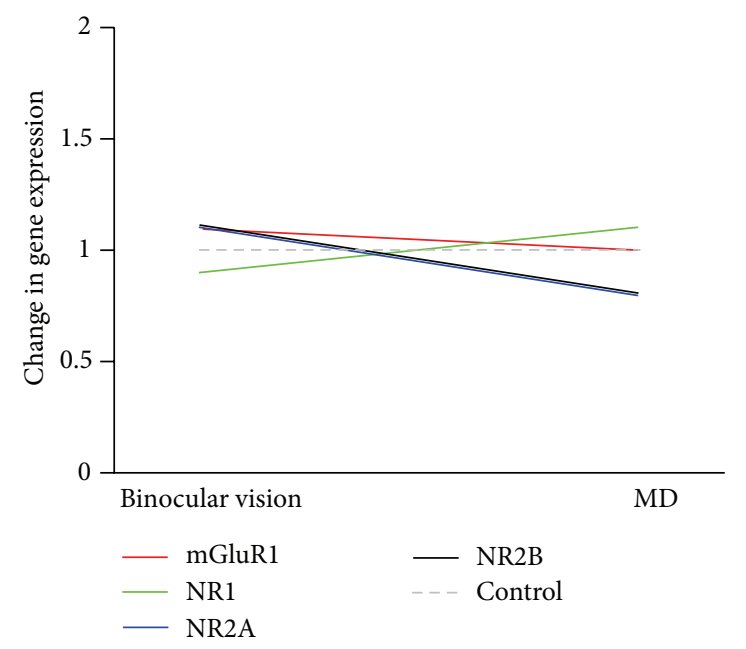

(b) Excitatory neurotransmission

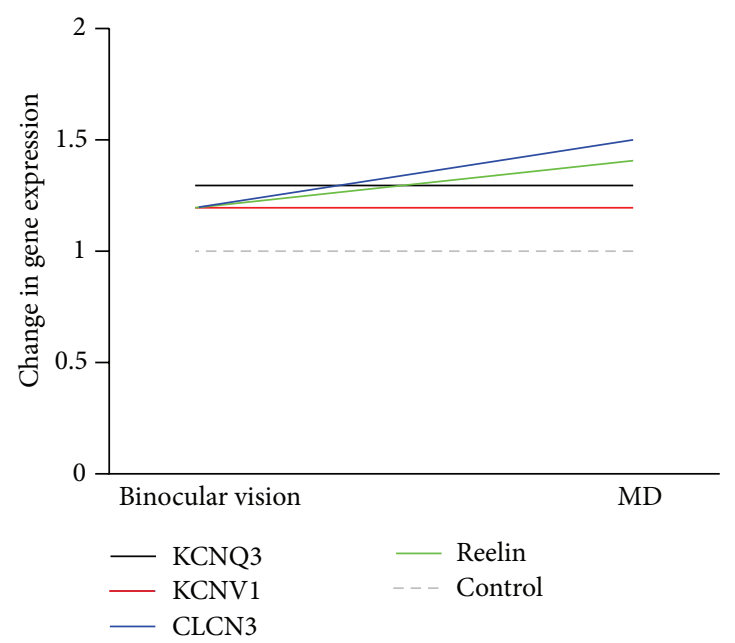

(d) Synaptic plasticity

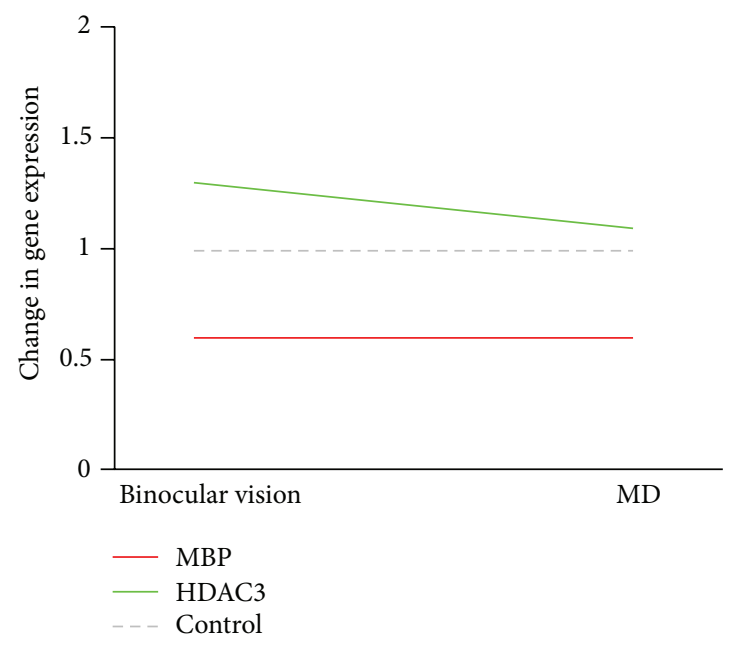

(f) Chromatin remodeling and myelination

FIGURE 2: Effects of fluoxetine in the expression of genes involved in critical period plasticity. qRT-PCR mRNA fold change comparison between the effects of fluoxetine in rats with binocular vision and monocular deprivation. Statistical data is grouped by binocular vision or monocular deprivation, and in each part the position of the line represents the fold change of the fluoxetine treated group (coloured line) with respect to the saline treated group (grey dashed line) (BV-Sal versus BV-Flx and MD-Sal versus MD-Flx, resp.). All gene expression was normalized using GAPDH as a control gene. 
3.2.6. Chromatin Remodeling and Myelination. Changes in the expression of transcripts that encode an enzyme that regulate chromatin susceptibility to transcription were detected in animals with binocular vision after chronic fluoxetine treatment. In particular, we found that $\mathrm{Hdac} 3$ expression was enhanced (Figure 2(f); $30 \%$ increased $P=0.02$ ). On the other hand, the expression of MBP, which encodes a basic protein of myelin, a repressive factor for visual cortex plasticity [33], was significantly reduced by fluoxetine treatment in animals with both binocular vision ( $40 \%$ decreased; $P=0.01)$ and monocular deprivation ( $40 \%$ decreased; $P=0.001)$.

\section{Discussion}

This study provides a large-scale analysis of changes in patterns of gene expression associated with the reopening of the critical period of plasticity in the adult visual system induced by the combination of fluoxetine treatment and monocular deprivation. Our findings suggest a scenario where an enhanced serotoninergic transmission induced by long-term fluoxetine treatment induces a shift of the inhibitory-excitatory balance $[8,29]$, which in turn promotes an alteration in the expression of genes involved in different biological themes that may underlie the functional modifications in the adult visual cortex related with the reopening of the critical period plasticity [40].

Our results reveal that the process of plasticity reactivation in adulthood involves both (i) a transient activation of neural mechanisms normally present during early stages of brain development and (ii) a removal of molecular factors that inhibit plasticity in adulthood [19]. Gene expression patterns involved in processes of synaptic plasticity, neuronal differentiation, and outgrowth were, indeed, differentially regulated by chronic fluoxetine treatment.

The increased expression of Reelin may represent an example of the first mechanism. Reelin is an extracellular glycoprotein involved in the migration and correct development of the cerebral cortex [41, 42]. Reelin is highly expressed by Cajal-Retzius neurons during development, but its expression is limited to a subpopulation of interneurons during the adulthood $[43,44]$. Although the function of Reelin in adult neurons remains unclear, its overexpression has been shown to enhance plasticity and learning, affecting presynaptic transmission [34, 45]. Our results demonstrate an upregulation of Reelin after chronic fluoxetine treatment, suggesting that the overexpression of molecules involved in the juvenile plasticity plays an important role in the reopening of the critical periods during the adulthood.

The proteolytic enzyme Mmp2, on the other hand, may drive mechanisms of synaptic plasticity by degrading extracellular matrix components that are inhibitory for plasticity, as observed in the adult hippocampus [39]. Increase of $M m p 2$ expression, indeed, was paralleled by a decrease of $M b p$ : a basic component of myelin, which is a repressive factor for visual cortex plasticity [33]. Our analysis of gene expression points towards a downregulation of $\mathrm{Mbp}$ following long-term antidepressant treatment, supporting the hypothesis that the removal of factors that are inhibitory for plasticity may provide a permissive environment for structural and functional changes of neuronal circuitries in the adult nervous system [19].

Chronic fluoxetine administration has been shown to promote structural changes in both excitatory $[46,47]$ and inhibitory circuits [48-50]. Although there is evidence that long-term fluoxetine administration promotes a reduction of GABA-mediated inhibition in adult visual cortical circuitries $[8,29]$, a compensatory mechanism might explain the increase in the expression of VGAT or GABRA4 that we observe in our experiment. These results are also in agreement with previous studies, in which fluoxetine treatment in combination with monocular deprivation produces an increase in the elongation of the tips of interneuronal dendrites [50], supporting the idea that inhibitory neurotransmission plays a key role in the reopening of the critical periods [20,22, 23]. Similarly, the change of NMDA receptor subunit composition, evidenced by the decrease in NMDA2A gene expression following antidepressant treatment, is particularly interesting in this respect. The expression of the NR2A subunit has been correlated with a progressive decrease of NMDA receptor currents during development $[51,52]$. This raises the possibility that a decrement of the NR2A/B ratio may increase NMDA receptors sensitivity thus causing the strengthening of synapses required for the potentiation of the nondeprived input [53].

Another highly significant notion that emerges from our data is that the changes promoted by the combination of fluoxetine with monocular deprivation, regarding the expression of transcription factors and proteins of the extracellular matrix, are opposed to those promoted by fluoxetine alone. This indicates that these molecules might be underlying the structural plasticity changes driven by monocular deprivation to produce the shift in the ocular dominance and its consolidation in the visual system [54].

Our findings support the hypothesis that the therapeutic effect of antidepressant drugs is dependent on changes in neuronal plasticity $[55,56]$. Importantly, these results open up new insights into the understanding of the mechanisms underlying the reopening of the critical period in the adult brain, by providing the basis of gene expression patterns for a visual deprivation paradigm that demonstrates the ability of the nervous system to translate environmental stimuli into structural and functional changes of neural circuitries.

\section{Authors' Contribution}

Ettore Tiraboschi and Ramon Guirado contributed equally to this work.

\section{References}

[1] L. C. Katz and C. J. Shatz, "Synaptic activity and the construction of cortical circuits," Science, vol. 274, no. 5290, pp. 1133-1138, 1996.

[2] N. Berardi, T. Pizzorusso, and L. Maffei, "Critical periods during sensory development," Current Opinion in Neurobiology, vol. 10, no. 1, pp. 138-145, 2000.

[3] V. B. Mountcastle, "The columnar organization of the neocortex," Brain, vol. 120, part 4, pp. 701-722, 1997. 
[4] D. Tropea, A. van Wart, and M. Sur, "Molecular mechanisms of experience-dependent plasticity in visual cortex," Philosophical Transactions of the Royal Society B, vol. 364, no. 1515, pp. 341355, 2009.

[5] D. H. Hubel and T. N. Wiesel, "The period of susceptibility to the physiological effects of unilateral eye closure in kittens," Journal of Physiology, vol. 206, no. 2, pp. 419-436, 1970.

[6] T. N. Wiesel and D. H. Hubel, "Single-cell responses in striate cortex of kittens deprived of vision in one eye," Journal of Neurophysiology, vol. 26, pp. 1003-1017, 1963.

[7] T. K. Hensch, "Critical period plasticity in local cortical circuits," Nature Reviews Neuroscience, vol. 6, no. 11, pp. 877-888, 2005.

[8] J. F. Maya-Vetencourt, A. Sale, A. Viegi et al., "The antidepressant fluoxetine restores plasticity in the adult visual cortex," Science, vol. 320, no. 5874, pp. 385-388, 2008.

[9] N. N. Karpova, A. Pickenhagen, J. Lindholm et al., "Fear erasure in mice requires synergy between antidepressant drugs and extinction training," Science, vol. 334, no. 6063, pp. 1731-1734, 2011.

[10] C. Orlando, J. Ster, U. Gerber, J. W. Fawcett, and O. Raineteau, "Perisynaptic chondroitin sulfate proteoglycans restrict structural plasticity in an integrin-dependent manner," The Journal of Neuroscience, vol. 32, Article ID 18017a, pp. 18009-18017, 2012.

[11] T. Pizzorusso, P. Medini, N. Berardi, S. Chierzi, J. W. Fawcett, and L. Maffei, "Reactivation of ocular dominance plasticity in the adult visual cortex," Science, vol. 298, no. 5596, pp. 12481251, 2002.

[12] L. Baroncelli, A. Sale, A. Viegi et al., "Experience-dependent reactivation of ocular dominance plasticity in the adult visual cortex," Experimental Neurology, vol. 226, no. 1, pp. 100-109, 2010.

[13] A. Sale, J. F. Maya-Vetencourt, P. Medini et al., "Environmental enrichment in adulthood promotes amblyopia recovery through a reduction of intracortical inhibition," Nature Neuroscience, vol. 10, no. 6, pp. 679-681, 2007.

[14] A. Sale, N. Berardi, and L. Maffei, "Enrich the environment to empower the brain," Trends in Neurosciences, vol. 32, no. 4, pp. 233-239, 2009.

[15] M. Scali, L. Baroncelli, M. C. Cenni, A. Sale, and L. Maffei, "A rich environmental experience reactivates visual cortex plasticity in aged rats," Experimental Gerontology, vol. 47, no. 4, pp. 337-341, 2012.

[16] M. Spolidoro, L. Baroncelli, E. Putignano, J. F. Maya-Vetencourt, A. Viegi, and L. Maffei, "Food restriction enhances visual cortex plasticity in adulthood," Nature Communications, vol. 2, no. 1, article 320, 2011.

[17] J. F. Maya-Vetencourt, E. Tiraboschi, D. Greco et al., "Experience-dependent expression of NPAS4 regulates plasticity in adult visual cortex," The Journal of Physiology, vol. 590, pp. 47774787, 2012.

[18] S. Miyata, Y. Komatsu, Y. Yoshimura, C. Taya, and H. Kitagawa, "Persistent cortical plasticity by upregulation of chondroitin 6sulfation," Nature Neuroscience, vol. 15, no. 3, pp. 414-422, 2012.

[19] D. Bavelier, D. M. Levi, R. W. Li, Y. Dan, and T. K. Hensch, "Removing brakes on adult brain plasticity: from molecular to behavioral interventions," The Journal of Neuroscience, vol. 30, no. 45, pp. 14964-14971, 2010.

[20] M. Beurdeley, J. Spatazza, H. H. C. Lee et al., "Otx2 binding to perineuronal nets persistently regulates plasticity in the mature visual cortex," The Journal of Neuroscience, vol. 32, pp. 94299437, 2012.
[21] H. Morishita, J. M. Miwa, N. Heintz, and T. K. Hensch, "Lynx1, a cholinergic brake, limits plasticity in adult visual cortex," Science, vol. 330, no. 6008, pp. 1238-1240, 2010.

[22] Z. J. Huang, A. Kirkwood, T. Pizzorusso et al., "BDNF regulates the maturation of inhibition and the critical period of plasticity in mouse visual cortex," Cell, vol. 98, no. 6, pp. 739-755, 1999.

[23] D. G. Southwell, R. C. Froemke, A. Alvarez-Buylla, M. P. Stryker, and S. P. Gandhi, "Cortical plasticity induced by inhibitory neuron transplantation," Science, vol. 327, no. 5969, pp. 1145$1148,2010$.

[24] G. Di Cristo, B. Chattopadhyaya, S. J. Kuhlman et al., "Activitydependent PSA expression regulates inhibitory maturation and onset of critical period plasticity," Nature Neuroscience, vol. 10, no. 12, pp. 1569-1577, 2007.

[25] T. A. Pham, S. Impey, D. R. Storm, and M. P. Stryker, "Cremediated gene transcription in neocortical neuronal plasticity during the developmental critical period," Neuron, vol. 22, no. 1, pp. 63-72, 1999.

[26] E. Putignano, G. Lonetti, L. Cancedda et al., "Developmental downregulation of histone posttranslational modifications regulates visual cortical plasticity," Neuron, vol. 53, no. 5, pp. 747759, 2007.

[27] Y. Wang, Q. Gu, and M. S. Cynader, "Blockade of serotonin-2C receptors by mesulergine reduces ocular dominance plasticity in kitten visual cortex," Experimental Brain Research, vol. 114, no. 2, pp. 321-328, 1997.

[28] Q. Gu and W. Singer, "Involvement of serotonin in developmental plasticity of kitten visual cortex," The European Journal of Neuroscience, vol. 7, no. 6, pp. 1146-1153, 1995.

[29] J. F. Maya-Vetencourt, E. Tiraboschi, M. Spolidoro, E. Castrén, and L. Maffei, "Serotonin triggers a transient epigenetic mechanism that reinstates adult visual cortex plasticity in rats," The European Journal of Neuroscience, vol. 33, no. 1, pp. 49-57, 2011.

[30] K. J. Livak and T. D. Schmittgen, "Analysis of relative gene expression data using real-time quantitative PCR and the 2$\Delta \Delta$ CT method," Methods, vol. 25, no. 4, pp. 402-408, 2001.

[31] M. Fagiolini and T. K. Hensch, "Inhibitory threshold for criticalperiod activation in primary visual cortex," Nature, vol. 404, no. 6774, pp. 183-186, 2000.

[32] T. K. Hensch, M. Fagiolini, N. Mataga, M. P. Stryker, S. Baekkeskov, and S. F. Kash, "Local GABA circuit control of experience-dependent plasticity in developing visual cortex," Science, vol. 282, no. 5393, pp. 1504-1508, 1998.

[33] A. W. McGee, Y. Yang, Q. S. Fischer, N. W. Daw, and S. H. Strittmatter, "Neuroscience: experience-driven plasticity of visual cortex limited by myelin and nogo receptor," Science, vol. 309, no. 5744, pp. 2222-2226, 2005.

[34] E. J. Weeber, U. Beffert, C. Jones et al., "Reelin and ApoE receptors cooperate to enhance hippocampal synaptic plasticity and learning," The Journal of Biological Chemistry, vol. 277, no. 42, pp. 39944-39952, 2002.

[35] T. M. Reeves, M. L. Prins, J. Zhu, J. T. Povlishock, and L. L. Phillips, "Matrix metalloproteinase inhibition alters functional and structural correlates of deafferentation-induced sprouting in the dentate gyrus," The Journal of Neuroscience, vol. 23, no. 32, pp. 10182-10189, 2003.

[36] J. Zuo, T. A. Ferguson, Y. J. Hernandez, W. G. Stetler-Stevenson, and D. Muir, "Neuronal matrix metalloproteinase-2 degrades and inactivates a neurite- inhibiting chondroitin sulfate proteoglycan," The Journal of Neuroscience, vol. 18, no. 14, pp. 52035211, 1998. 
[37] V. W. Yong, C. Power, P. Forsyth, and D. R. Edwards, "Metalloproteinases in biology and pathology of the nervous system," Nature Reviews Neuroscience, vol. 2, no. 7, pp. 502-511, 2001.

[38] V. Nagy, O. Bozdagi, A. Matynia et al., "Matrix metalloproteinase- 9 is required for hippocampal late-phase long-term potentiation and memory," The Journal of Neuroscience, vol. 26, no. 7, pp. 1923-1934, 2006.

[39] S. E. Meighan, P. C. Meighan, P. Choudhury et al., "Effects of extracellular matrix-degrading proteases matrix metalloproteinases 3 and 9 on spatial learning and synaptic plasticity," Journal of Neurochemistry, vol. 96, no. 5, pp. 1227-1241, 2006.

[40] J. F. Maya-Vetencourt and N. Origlia, "Visual cortex plasticity: a complex interplay of genetic and environmental influences," Neural Plasticity, vol. 2012, Article ID 631965, 14 pages, 2012.

[41] J. Herz and Y. Chen, "Reelin, lipoprotein receptors and synaptic plasticity," Nature Reviews Neuroscience, vol. 7, no. 11, pp. 850859, 2006.

[42] G. D’Arcangelo, G. G. Miao, S.-C. Chen, H. D. Soares, J. I. Morgan, and T. Curran, "A protein related to extracellular matrix proteins deleted in the mouse mutant reeler," Nature, vol. 374, no. 6524, pp. 719-723, 1995.

[43] S. H. Fatemi, "Reelin glycoprotein: structure, biology and roles in health and disease," Molecular Psychiatry, vol. 10, no. 3, pp. 251-257, 2005.

[44] E. Soriano and J. A. del Río, "The cells of cajal-retzius: still a mystery one century after," Neuron, vol. 46, no. 3, pp. 389-394, 2005.

[45] S. Hellwig, I. Hack, J. Kowalski et al., "Role for reelin in neurotransmitter release," The Journal of Neuroscience, vol. 31, no. 7, pp. 2352-2360, 2011.

[46] T. Hajszan, N. J. MacLusky, and C. Leranth, "Short-term treatment with the antidepressant fluoxetine triggers pyramidal dendritic spine synapse formation in rat hippocampus," The European Journal of Neuroscience, vol. 21, no. 5, pp. 1299-1303, 2005.

[47] R. Guirado, E. Varea, E. Castillo-Gómez et al., "Effects of chronic fluoxetine treatment on the rat somatosensory cortex: activation and induction of neuronal structural plasticity," Neuroscience Letters, vol. 457, no. 1, pp. 12-15, 2009.

[48] S. H. Fatemi, T. J. Reutiman, and T. D. Folsom, "Chronic psychotropic drug treatment causes differential expression of Reelin signaling system in frontal cortex of rats," Schizophrenia Research, vol. 111, no. 1-3, pp. 138-152, 2009.

[49] R. Guirado, D. Sanchez-Matarredona, E. Varea, C. Crespo, J. M. Blasco-Ibáñez, and J. Nacher, "Chronic fluoxetine treatment in middle-aged rats induces changes in the expression of plasticity-related molecules and in neurogenesis," BMC Neuroscience, vol. 13, no. 1, article 5, 2012.

[50] J. L. Chen, W. C. Lin, J. W. Cha, P. T. So, Y. Kubota, and E. Nedivi, "Structural basis for the role of inhibition in facilitating adult brain plasticity," Nature Neuroscience, vol. 14, no. 5, pp. 587-596, 2011.

[51] A. C. Flint, U. S. Maisch, J. H. Weishaupt, A. R. Kriegstein, and H. Monyer, "NR2A subunit expression shortens NMDA receptor synaptic currents in developing neocortex," The Journal of Neuroscience, vol. 17, no. 7, pp. 2469-2476, 1997.

[52] G. Carmignoto and S. Vicini, "Activity-dependent decrease in NMDA receptor responses during development of the visual cortex," Science, vol. 258, no. 5084, pp. 1007-1011, 1992.

[53] K. K. A. Cho, L. Khibnik, B. D. Philpot, and M. F. Bear, "The ratio of NR2A/B NMDA receptor subunits determines the qualities of ocular dominance plasticity in visual cortex," Proceedings of the National Academy of Sciences of the United States of America, vol. 106, no. 13, pp. 5377-5382, 2009.

[54] A. W. Lyckman, S. Horng, C. A. Leamey et al., "Gene expression patterns in visual cortex during the critical period: synaptic stabilization and reversal by visual deprivation," Proceedings of the National Academy of Sciences of the United States of America, vol. 105, no. 27, pp. 9409-9414, 2008.

[55] E. Castrén and T. Rantamäki, "The role of BDNF and its receptors in depression and antidepressant drug action: reactivation of developmental plasticity," Developmental Neurobiology, vol. 70, no. 5, pp. 289-297, 2010.

[56] N. Tsankova, W. Renthal, A. Kumar, and E. J. Nestler, "Epigenetic regulation in psychiatric disorders," Nature Reviews Neuroscience, vol. 8, no. 5, pp. 355-367, 2007. 

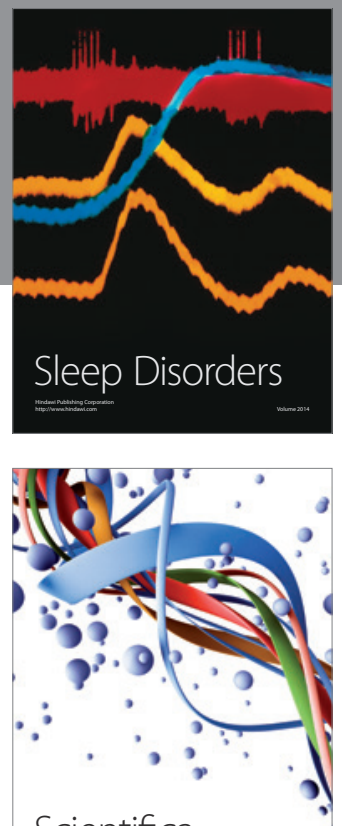

Scientifica
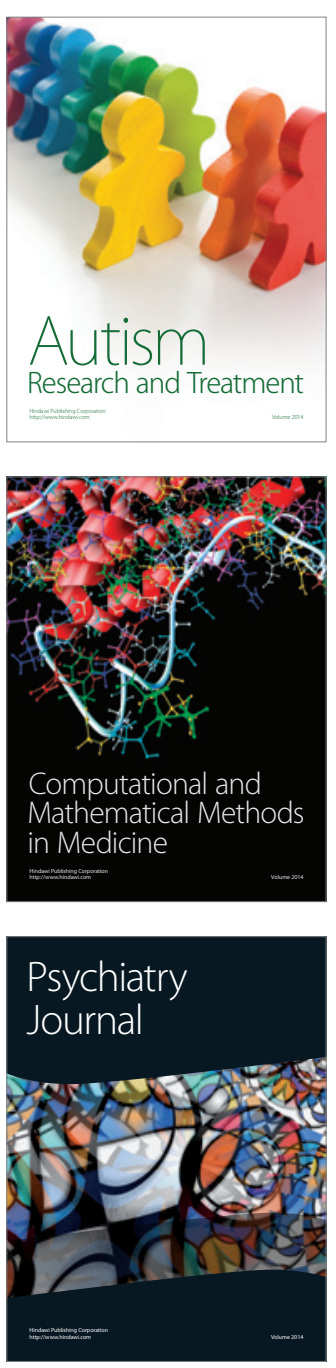
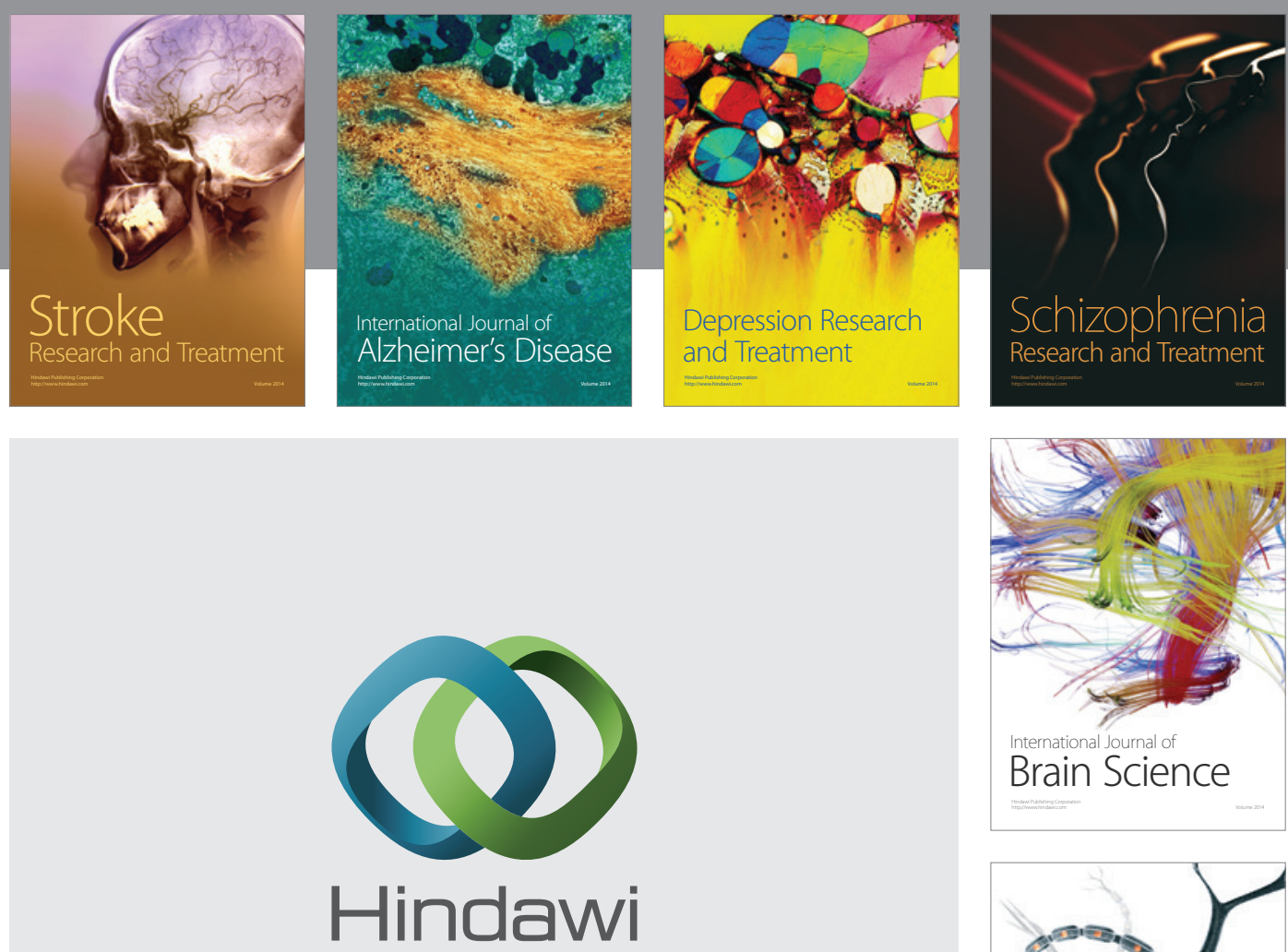

Submit your manuscripts at

http://www.hindawi.com
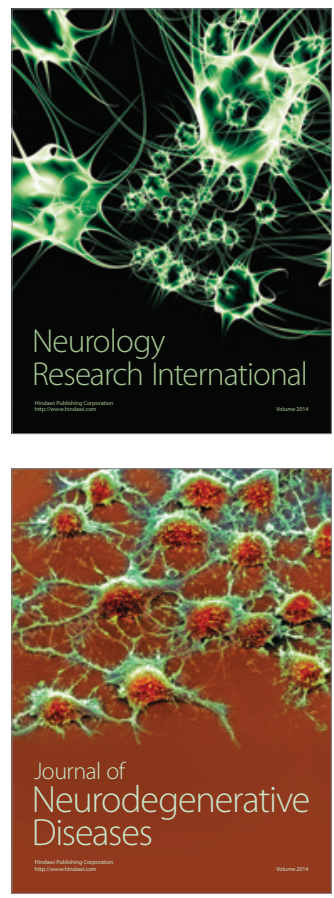

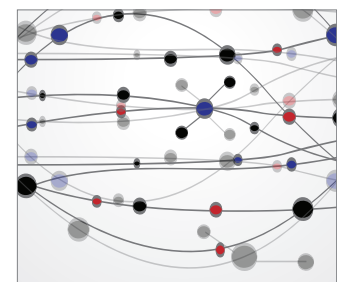

The Scientific World Journal
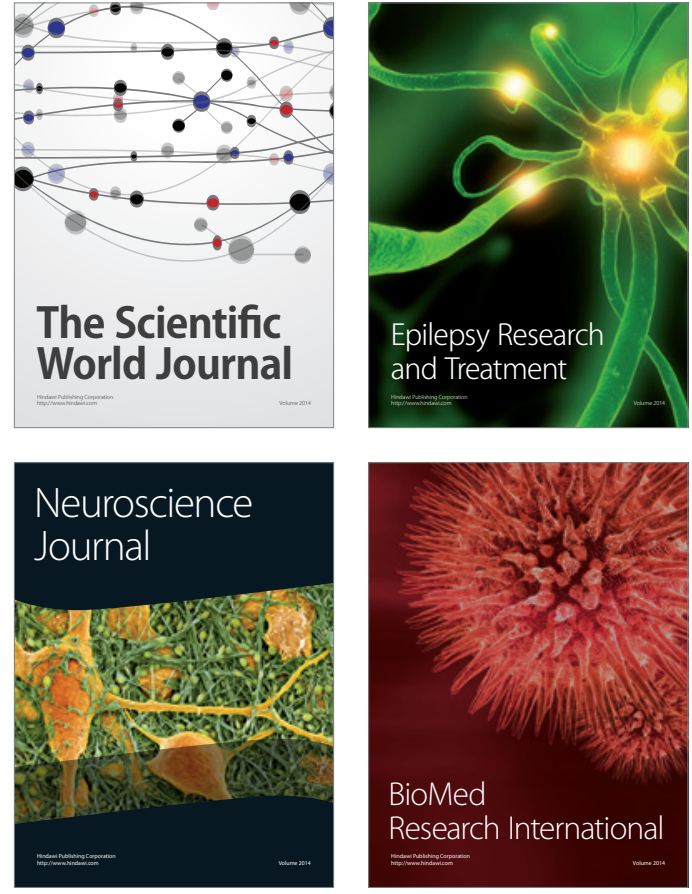

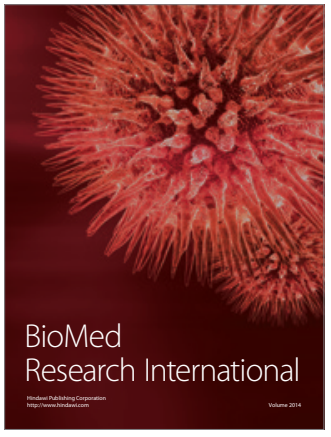

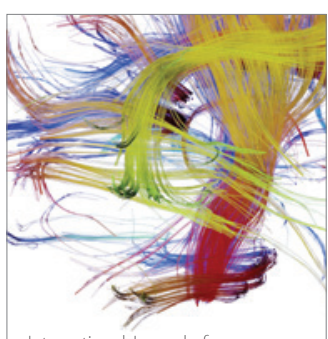

Brain Science

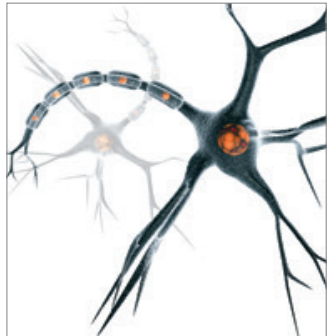

Neural Plasticity
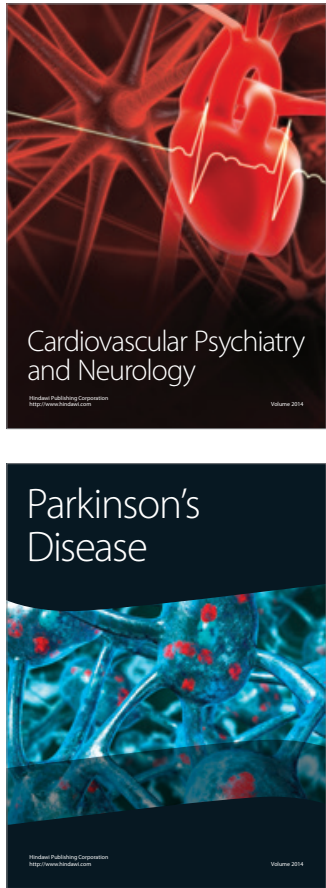